\title{
DVD+RW: 2-WAY COMPATIBILITY FOR VIDEO AND DATA APPLICATIONS
}

\author{
Chris Buma, Robert Brondijk, Sorin G. Stan ${ }^{2}$ \\ ${ }^{1}$ Philips Consumer Electronics, The Netherlands \\ ${ }^{2}$ Philips Optical Storage, The Netherlands
}

\begin{abstract}
This paper discusses the 4.7-GB Digital Versatile Disc plus ReWritable $(D V D+R W)$ system as a future replacement for the Video Cassette Recorder (VCR). It is shown that Digital Versatile Disc (DVD) discs can be recorded in a fully compatible manner with the existing DVD-Video standard and players. The compatibility issue is addressed from the perspective of the DVD-Video physical and logical formats.
\end{abstract}

\section{INTRODUCTION}

Since its market introduction in 1996, the Digital Versatile Disc has experienced a tremendous sales boost, with more and more people discovering the advantages of this highcapacity optical disc medium. DVD-Video, in particular, has attracted the public attention due to the superb audio and video quality it offers. Recordable DVD systems, on the other hand, have just entered the race for obtaining a status on the consumer electronics market.

One of the key features of a recordable DVD system must be its backward compatibility with the existing DVDVideo players. Several systems have been proposed, some of them making use of the Real-Time Video (RTV) recording specifications [4]. However, the Digital Versatile Disc plus Rewritable (DVD+RW) employs a different recording model while the recorded disc remains fully compatible with the existing standards $[1,2,3]$. This feature should be seen as the ability of the existing DVD-Video equipment and DVD-ROM drives to play back the DVD+RW media, irrespective of the type and brand of the DVD+RW recorder on which the disc was recorded. This 2-way compatibility on both the video consumer electronics and computer markets is illustrated in Fig. 1.

To achieve the compatibility depicted in Fig. 1, several issues related to the physical disc format and the recording technique had to be solved.

The correspondence should be addressed to Sorin G. Stan, Philips Optical Storage, Bldg. SFJ-1, P.O. Box 80002, 5600 JB Eindhoven, The Netherlands. E-mail: sorin.stan@ieee.org

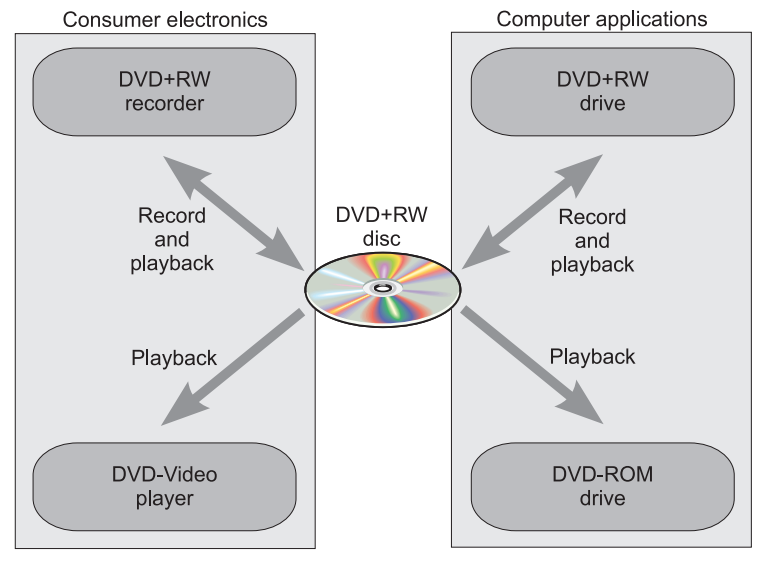

Figure 1 Playback and recording compatibility of the DVD+RW discs on the existing consumer markets.

\section{PHYSICAL COMPATIBILITY}

From the viewpoint of the physical disc format, a similar compatibility issue was previously solved for the Compact Disc Recordable (CD-R) and Compact Disc Rewritable (CD-RW) media. The DVD+RW disc uses the same phasechange recording materials as CD-RW (Ag-In-Sb-Te alloys, for example) and it also employs a wobbled groove arranged in a continuous spiral, which extends from the inner to the outer disc radius. Data is recorded as a series of marks embedded in the phase-change layer of the undulated groove.

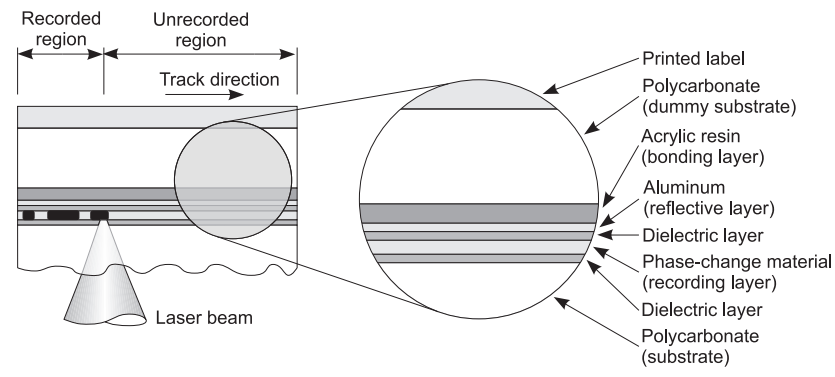

Figure 2 Cross section through a DVD+RW disc. 
The groove, which does not disturb the readout process in read-only DVD systems, provides means for (i) radial tracking, (ii) addressing any location on the blank disc to correctly position the recording laser spot, and (iii) exact synchronization and alignment between old data blocks on disc and data to be newly written. The latter two issues are discussed with more details in [5].

The compatibility between DVD+RW and DVD-Video (or DVD-ROM) discs has been achieved by preserving the essential physical parameters [1] of the existing read-only media. An overview of several parameters is given in Table 1. It is important to remark that, due to the phase-change material, the DVD+RW reflectivity is equal to the reflectivity of the dual-layer read-only DVD disc. For the sake of comparison, recall that CD-RW media also exhibit lower reflectivity than read-only CDs.

Another physical compatibility issue regards the continuity of the data stream on the DVD+RW disc. Basically, it is always possible to insert a new data block between two existent ones as depicted in Fig. 3. Dummy areas are needed to account for the limited precision of inserting new data. However, the DVD+RW format allows for "lossless" linking, whereby bit-accurate positioning of the new data stream can be achieved [5]. This technique is illustrated in Fig. 4. Clearly, lossless recording leads to a disc which is compatible with the existing DVD-Video and DVD-ROM media.

\begin{tabular}{|c|c|c|c|}
\hline \multirow{2}{*}{ Parameter } & \multirow{2}{*}{ DVD+RW } & \multicolumn{2}{|c|}{ DVD-Video, DVD-ROM } \\
\hline & & single layer & dual layer \\
\hline Wavelength [nm] & \multicolumn{3}{|c|}{650} \\
\hline NA (player) & \multicolumn{3}{|c|}{0.6} \\
\hline Capacity $\left[\times 10^{9}\right.$ bytes $]$ & 4.70 & 4.70 & 8.54 \\
\hline Track pitch $[\mu \mathrm{m}]$ & \multicolumn{3}{|c|}{0.74} \\
\hline Min. mark lenth $[\mu \mathrm{m}]$ & 0.4 & 0.4 & 0.44 \\
\hline Reflectivity [\%] & $18 \ldots 30$ & $45 \ldots 85$ & $18 \ldots 30$ \\
\hline Track-cross modul. [\%] & \multicolumn{3}{|c|}{$>10$} \\
\hline RF modulation & \multicolumn{3}{|c|}{$>0.6$} \\
\hline Asymmetry & \multicolumn{3}{|c|}{$-0.05 \ldots 0.15$} \\
\hline Bottom jitter [\%] & $<9$ & $<8$ & $<8$ \\
\hline Thickness [mm] & $0.58 \ldots 0.62$ & $0.57 \ldots 0.63$ & $0.57 \ldots 0.63$ \\
\hline Radial deviation [deg.] & 0.7 & 0.8 & 0.8 \\
\hline
\end{tabular}

Table 1 Several physical parameters of the DVD+RW, DVD-Video, and DVD-ROM discs.

\section{LOGICAL COMPATIBILITY}

The DVD-Video standard also includes specifications for the file system [2] as well as for the video format [3]. At this logical level, the compatibility is guaranteed by making use of a tape model while recording. As depicted in Fig. 5, this model allows for appending or insertion of a new video sequence, erasing the previously recorded video in the latter case. The tape model employs two recording sequences:

\begin{tabular}{|c|c|c|c|}
\hline \multicolumn{2}{|c|}{ Old recording } & \multicolumn{2}{|c|}{ New recording } \\
\hline $32 \mathrm{kB}$ & $32 \mathrm{kB}$ & $30 \mathrm{kB}$ & $32 \mathrm{kB}$ \\
\hline & Nominal data block & $\begin{array}{l}\text { Link data block } \\
\text { nking region }(2 \mathrm{k}\end{array}$ & \\
\hline
\end{tabular}

Figure 3 DVD recording with insertion of linking areas between old and new data blocks.

\begin{tabular}{|c|c|c|c|}
\hline \multicolumn{2}{|c|}{ Old recording } & \multicolumn{2}{|c|}{ New recording } \\
\hline $32 \mathrm{kB}$ & $32 \mathrm{kB}$ & $32 \mathrm{kB}$ & $32 \mathrm{kB}$ \\
\hline
\end{tabular}

Figure 4 Bit-accurate DVD recording using DVD+RW disc format.

\begin{tabular}{|c|c|c|c|c|c|c|c|}
\hline $\begin{array}{c}\text { Control } \\
\text { data }\end{array}$ & $\begin{array}{c}\text { Previous file } \\
\text { system }\end{array}$ & $\begin{array}{c}\text { Previous DVD } \\
\text { control files }\end{array}$ & $\begin{array}{c}\text { Old } \\
\text { video }\end{array}$ & \begin{tabular}{|c|} 
New \\
video
\end{tabular} & $\begin{array}{c}\text { Old } \\
\text { video }\end{array}$ & $\begin{array}{c}\text { Backup } \\
\text { control files }\end{array}$ & $\begin{array}{c}\text { Lead } \\
\text { out }\end{array}$ \\
\hline
\end{tabular}

Figure 5 Double-pass insertion of a new video title according to the tape model.

during the first pass, the new video title is added, the DVD control files are backed up, and the lead out is written; during the second pass, the file system and the control files are updated. This double-sequence recording ensures the logical compatibility with the existing DVD standards $[2,3]$.

\section{CONCLUSION}

It has been shown that DVD+RW physical and logical disc formats fully comply with the existing read-only DVD specifications. It is therefore possible for the existing DVDVideo players and DVD-ROM drives to play back the newly developed DVD+RW disc, immediately after the latter has been recorded. Because the DVD-Video has already been successfully accepted by the consumer electronics market, it is expected that DVD+RW will replace the ubiquitous Video Cassette Recorder (VCR) by the end of this decade.

\section{REFERENCES}

[1] DVD Forum. DVD Specifications for Read-Only DiscPart 1. Physical Specifications. Version 1.01, 1997.

[2] DVD Forum. DVD Specifications for Read-Only DiscPart 2. File System Specifications. Version 1.01, 1997.

[3] DVD Forum. DVD Specifications for Read-Only DiscPart 3. Video Specifications. Version 1.1, 1997.

[4] DVD Forum. DVD Video Recording for Rewritable and Recordable Discs-Part 3. Video Recording. Version 1.0, 1999.

[5] van Vlerken, J.J.L.M. and de Kimpe, W. "Format Detection for DVD+ReWritable 4.7 GB". IEEE International Conference on Consumer Electronics. Digest of Technical Papers, June 13-15, 2000, Los Angeles, U.S.A. 